\title{
Phase-transition behavior of a crystalline polymer near the melting point: case studies of the ferroelectric phase transition of poly(vinylidene fluoride) and the $\beta$-to- $\alpha$ transition of trans-1,4-polyisoprene
}

\author{
Paramita Jaya Ratri and Kohji Tashiro
}

Many crystalline polymers exhibit phase transitions between the low- and high-temperature phases during the heating process. Sometimes, however, the temperature-dependent measurement of the X-ray diffraction peaks and vibrational spectra leads us to the incorrect conclusion that these two phases transform in a direct solid-to-solid transition mode instead of the melt and recrystallization process. As a reliable method to confirm the transition behavior just below the melting region, we have developed a temperature-jump cell system, with which a time-dependent measurement of the X-ray diffraction peaks (and Fourier-transform infrared spectra) is performed in the rapidly changing temperature process from the low- to the hightemperature phase. This method was applied to two types of polymers and revealed that (i) the high-temperature phase transition of poly(vinylidene fluoride) is not a direct ferroelectric transition between the ferroelectric and paraelectric phases, as proposed in our previous studies (Polymer (1983), Polym. Bull. (1983)), but a melt of the ferroelectric phase (form I) followed by the recrystallization into the nonpolar crystalline phase (form III or V) and (ii) the $\beta$-to- $\alpha$ phase transition of trans-1,4-polyisoprene had been also controversial for many years but it was revealed to occur via a melt-recrystallization mechanism.

Polymer Journal (2013) 45, 1107-1114; doi:10.1038/pj.2013.42; published online 1 May 2013

Keywords: melt-recrystallization; phase transition; poly(vinylidene fluoride); time-resolved FTIR spectral measurement; time-resolved X-ray diffraction measurement; trans-1,4-polyisoprene

\section{INTRODUCTION}

Crystalline polymers exhibit various types of crystalline forms depending on the sample-preparation conditions. These crystalline forms can exhibit phase transitions by changing the external conditions, for example, the thermally induced phase transitions of polytetrafluoroethylene, ${ }^{1,2}$ aliphatic nylons, ${ }^{3-6}$ isotactic polybutene-1 $1^{7,8}$ and so on, and the stress-induced transitions of poly(tetramethylene terephthalate) $)^{9,10}$ and poly(ethylene oxide). ${ }^{11}$ The temperature-dependent measurement of X-ray diffraction can provide insight into the phase-transition behavior. Unfortunately, some unsolved problems remain even after carefully performing temperature-dependent X-ray diffraction experiments. For example, vinylidene fluoride-trifluoroethylene (VDF-TrFE) copolymers are quite unique polymers because they exhibit a reversible ferroelectric phase transition between the ferroelectric low-temperature phase and the paraelectric high-temperature phase during the heating and cooling processes. ${ }^{12-14}$ Poly(vinylidene fluoride) (PVDF) form I, being an extrapolation to a VDF content of $100 \%$, is assumed to exhibit this reversible ferroelectric phase transition. The prediction based on the phase diagram produced from a series of VDF-TrFE copolymers was that the phase transition of PVDF form I should occur in the temperature region near the melting point. Actually, the ferroelectric-paraelectric phase transition was reported to occur immediately below the melting point. ${ }^{15,16}$ This reported observation has not been validated because a competing possibility of meltrecrystallization cannot be ignored. ${ }^{17,18}$ trans-1,4-polyisoprene (TPI; gutta percha) was also reported to exhibit the solid-state transition from the $\beta$ form to the $\alpha$ form by heating, ${ }^{19-21}$ but this observation has yet to be confirmed. ${ }^{22-25}$ A similar situation might exist for the other types of crystalline polymers.

Ambiguity remains regarding the phase transition of crystalline polymers. Which transition mechanism is correct between the following two manners: (i) the direct solid-to-solid phase transition or (ii) the crystal melts to an amorphous phase or it disorders to a phase similar to the liquid-crystalline phase, followed by recrystallization to another crystalline form (melt-recrystallization mechanism). In some cases, the apparently direct solid-to-solid phase transition, that is, mechanism (i) stated above, might not be the actual 
mechanism in play; rather, the phase-transition mechanism between these two solid phases can occur via an intermediate amorphous or liquid-crystalline phase, that is, mechanism (ii) stated above. Mistakenly, ignoring such an intermediate phase may lead us to the wrong conclusion regarding the phase-transition mechanism.

How can such a mistake occur in the clarification of the phasetransition behavior? Normally, we measure the X-ray diffraction and/ or infrared and Raman spectra during the course of the heating (and cooling) process. Unfortunately, the phase-transition region is not very narrow for general crystalline polymers, but it occurs over a relatively wide temperature region; in addition, the crystalline polymers do not exhibit very sharp and well-separated X-ray diffraction peaks. As a result, the existing phases overlap over a wide temperature region, and the signature of the phase-transition process becomes ambiguous, leading us to produce an incorrect conclusion regarding the phase-transition behavior.

We propose one way to avoid such a serious problem, which involves performing time-resolved measurement of the X-ray diffraction peaks and the Fourier-transform infrared (FTIR) spectra with high temporal resolution during the rapid temperature-jump process from the low-temperature phase region to the high-temperature phase region. During a steep temperature change, the individual phase may appear and disappear in sequence because of a slight difference in the existing temperature region. Correspondingly, the X-ray diffraction and vibrational bands of the phases are observed at slightly shifted times. The proposed method was applied to two concrete cases that had remained unresolved for a long time in spite of their significance in the structural science of polymers: the high-temperature phase transition of PVDF form I and the $\beta$-to- $\alpha$ phase transition of TPI. The reasons for choosing these two polymers are stated below.

PVDF form I is one of the most common ferroelectric polymers and exhibits a polar crystal structure consisting of the parallel array of $\mathrm{CF}_{2}$ dipoles of the planar-zigzag chains along the $b$ axis. ${ }^{26,27}$ Ferroelectric crystals generally exhibit a phase transition between the polar (ferroelectric) and nonpolar (paraelectric) phases during the heating (or cooling) process. ${ }^{14} \mathrm{~A}$ possible ferroelectric phase transition was investigated in detail for this polymer by referring to the experimental data measured for a series of VDF copolymers. We performed temperature-dependent measurements of the X-ray diffraction peaks and the infrared spectra during the heating process of a PVDF form I sample. ${ }^{15,16}$ The X-ray diffraction peaks and infrared bands intrinsic to the crystalline form III (or V) were detected in the temperature region immediately below the melting point. However, the possibility of a melt-recrystallization mechanism occurring in PVDF was suggested by others, ${ }^{17,18}$ so the existence of the ferroelectric phase transition has not been confirmed up to now, even though PVDF form I is one of the most common ferroelectric polymers.

A similar situation also applies to TPI. The TPI polymer exhibits several types of crystalline forms depending on the preparation conditions, among which the $\alpha$ - and $\beta$-forms are normally observed during the heating and cooling processes. Some reports proposed that the $\beta$-form transforms to the $\alpha$-form directly (solid-to-solid transition), ${ }^{19-21}$ but other reports proposed another possible phasetransformation mechanism involving the melting of the $\beta$ form followed by the recrystallization into the $\alpha$ form in a higher temperature region (melt-recrystallization). ${ }^{22-25}$

Determining the correct phase-transition mechanism for both PVDF and TPI may reveal a new aspect regarding the phase transition of polymers occurring near the melting region and may thereby become an important trigger to reinvestigate the phase-transition behaviors proposed for many crystalline polymers from a different point of view.

\section{EXPERIMENTAL PROCEDURE}

\section{Samples}

PVDF form I. Kynar, the PVDF sample, was supplied by Arkema (Elf Atochem Inc., Colombes, France). The melting point was approximately $167^{\circ} \mathrm{C}$, slightly lower than that of the KF series $\left(174^{\circ} \mathrm{C}\right)$ supplied by Kureha Co. Ltd., Tokyo, Japan. The sample was dissolved into the highly polar solvent hexamethylene phosphoric triamide at room temperature. ${ }^{28}$ This solution was cast onto a glass slide at an ambient temperature for 1 month. This quite slow evaporation of hexamethylene phosphoric triamide resulted in a highly crystalline and unoriented pure form I film.

TPI $\beta$-form. The synthetic TPI sample with $99 \%$ trans content was purchased from Polysciences Inc. (Warrington, PA, USA). As described in a previous study, ${ }^{29}$ and the unoriented pure $\beta$-form sample was prepared by quenching the melt at liquid $\mathrm{N}_{2}$ temperature followed by slowly heating to room temperature.

\section{Measurements}

Differential scanning calorimetry. A differential scanning calorimeter (DSC) TA DSC Q1000 was used to measure the DSC thermograms in a nitrogen gas atmosphere at a heating rate of $1{ }^{\circ} \mathrm{C} \mathrm{min}^{-1}$.

$X$-ray diffraction measurements. Wide-angle X-ray diffraction (WAXD) profiles of the unoriented samples were measured in the stepwise heating process using a Rigaku RINT-TTR III diffractometer (Rigaku, Tokyo, Japan) in a symmetric reflection mode. The incident X-ray beam was a graphite-monochromatized $\mathrm{Cu}-\mathrm{K}_{\alpha}(\lambda=1.5418 \AA)$ line, with an incident angle that was scanned over the diffraction angle $(2 \theta)$ range of $5-35^{\circ}$, and the diffracted $\mathrm{X}$-rays were detected using a two-dimensional semiconductor detector (D'Tex; Rigaku). During the X-ray diffraction measurement, the DSC thermogram was also measured simultaneously at a scanning rate of $1^{\circ} \mathrm{C} \mathrm{min}^{-1}$.

The WAXD measurements were performed in both the stepwise heating (i) and temperature-jump (ii) modes. (i) The stepwise heating experiments were performed using a Rigaku R-axis VII X-ray diffractometer equipped with a flat imaging plate as a detector set at a distance of $110 \mathrm{~mm}$ from the sample, where a graphite-monochromatized $\mathrm{Cu}-\mathrm{K}_{\alpha}$ line was used as an incident X-ray beam. (ii) In the temperature-jump process, the WAXD data were measured using a Rigaku Nanoviewer X-ray diffractometer. The sample was set in a Linkam temperature controller cell (Linkam, Surrey, UK). The temperature ramp rate was approximately $90^{\circ} \mathrm{C} \mathrm{min}{ }^{-1}$. The temperature of the sample was constantly monitored by inserting a thermocouple into a sample.

Fourier-transform infrared spectral measurements. The temperature- and time-dependent FTIR spectra were measured using a Varian FTS7000 rapidscan-type FTIR spectrometer (Varian (Agilent), Santa Clara, CA, USA) with an mercury cadmium telluride detector in transmission mode at a resolution power of $2 \mathrm{~cm}^{-1}$. The sample was set in a Linkam temperature controller cell with $\mathrm{KBr}$ windows (Japan Spectroscopic Co. Ltd., Tokyo, Japan).

Temperature-dependent viscoelastic measurement. The temperature dependence of the dynamic Young's modulus $\left(E^{\prime}, E^{\prime \prime}\right.$ and $\left.\tan (\delta)\right)$ was measured during the heating process of an unoriented TPI $\beta$-form sample. A dynamic viscoelastometer DVA-300 (IT Keisoku Seigyo Co., Osaka, Japan) was used to measure the viscoelastic parameters. The sample heating rate was $2{ }^{\circ} \mathrm{C} \mathrm{min}{ }^{-1}$, and the dynamic tensile frequency was $10 \mathrm{~Hz}$, using a maximal strain of $<1 \%$.

\section{RESULTS AND DISCUSSION}

\section{Phase transition of PVDF form I}

Figure 1 shows the previously proposed phase diagram of the VDF-TrFE copolymers with the various VDF contents. ${ }^{14}$ The temperature-dependent X-ray diffraction and infrared spectral measurements of an oriented PVDF form I sample indicated the transition to form III $^{30,31}$ (or form $\mathrm{V}^{32}$ ) with the TTTGTTTG conformation in the temperature region immediately below the melting point, as indicated by the solid circles in Figure 1. Because PVDF contains a small amount of head-to-head and tail-to-tail 


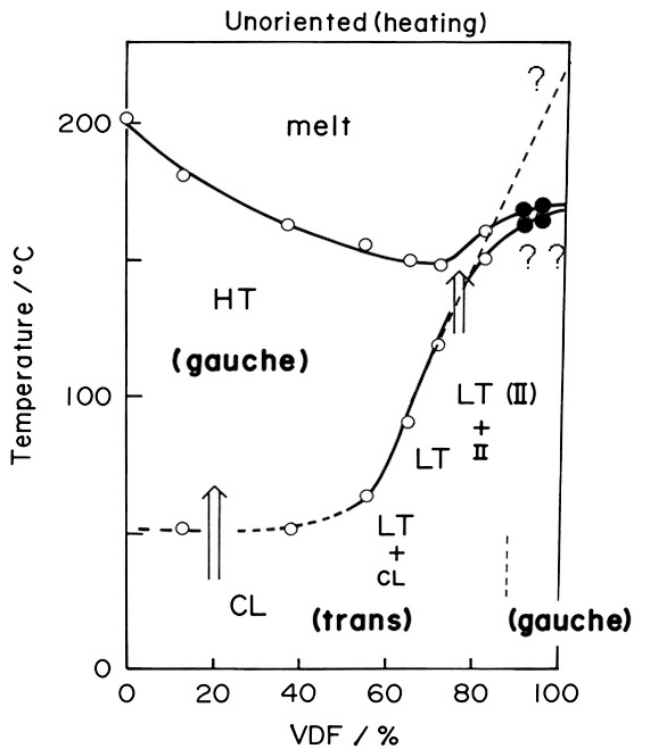

Figure 1 Ferroelectric phase-transition scheme of the VDF-TrFE copolymers. The solid circles correspond to the transitions revealed for PVDF form I (oriented sample) reported in previous studies. ${ }^{15,16} \mathrm{CL}$, cooled phase; HT, high-temperature phase; LT, low-temperature phase; II, crystalline form II similar to that of PVDF. ${ }^{14}$

abnormal linkages, the data were plotted as the copolymers with 90-95\% VDF content. These data points indicate that the ferroelectric phase transition of PVDF form I occurs just below the melting point. ${ }^{15,16}$ However, the melt-recrystallization phenomenon remains as another possible mechanism for the phase transition.

The PVDF sample utilized here was the hexamethylene phosphoric triamide-cast unoriented form I film. The time-resolved measurement was performed using a rapid-scanning FTIR spectrometer during the temperature-jump process, where the maximal heating rate from 105 to $150{ }^{\circ} \mathrm{C}$ was approximately $620^{\circ} \mathrm{C} \mathrm{min}{ }^{-1}$. The infrared spectra were measured every $10 \mathrm{~s}$ during the temperature-jump process. The spectral changes observed are shown in Figure 2: (a) 1000$1300 \mathrm{~cm}^{-1}$ and (b) $950-750 \mathrm{~cm}^{-1}$. During the heating process, the infrared bands of the form I crystal were detected at $840,1275 \mathrm{~cm}^{-1}$ and so on up to $155^{\circ} \mathrm{C}, 33$ at which point the temperature was fixed and the time-dependent measurement was performed to observe the spectral changes during the isothermal heating process. Approximately $10 \mathrm{~min}$ later, new bands started to appear at 808 , 831 and $1240 \mathrm{~cm}^{-1}$, which correspond to the infrared bands intrinsic to the form III conformation. ${ }^{28,31}$

In Figure 3, the integrated spectral intensity was plotted against time for the infrared bands of forms I $\left(840 \mathrm{~cm}^{-1}\right)$ and III $\left(831 \mathrm{~cm}^{-1}\right)$, as well as the band of the amorphous phase at $1109 \mathrm{~cm}^{-1}$. Immediately after the temperature reached $155^{\circ} \mathrm{C}$, the form I band intensity decreased dramatically and the amorphous band intensity increased, clearly indicating the melting of the form I crystal. Approximately $10 \mathrm{~min}$ later, the form III band began to appear, and the amorphous band decreased, indicating that the crystalline form III (or another form with the same chain conformation of TTTGTTTG $\overline{\text { ) }}$ crystallized from the melt. The other possible forms to form III are the alternative packing structures of the molecular chains with TTTGTTTG form, for example, the crystalline form $\mathrm{V}$ or the nonpolar form III. ${ }^{32}$

The data presented above confirmed that during the temperature jump, crystalline form I initially melted at $155^{\circ} \mathrm{C}$, followed by recrystallization into crystalline form III (or form V). As mentioned a

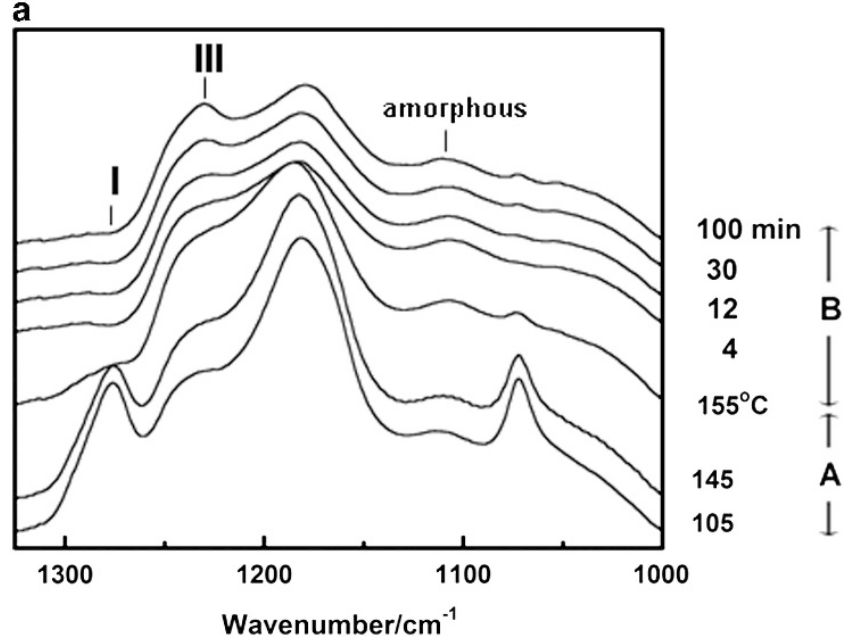

b

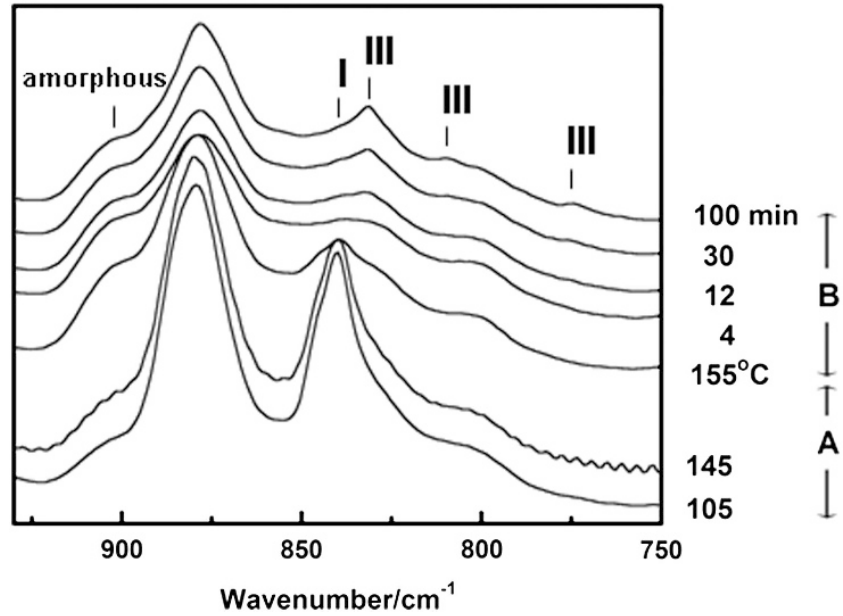

Figure 2 Time dependence of the infrared spectra of PVDF form I measured in the isothermal heating process. (a) The frequency region of $1000-1300 \mathrm{~cm}^{-1}$ and (b) $950-750 \mathrm{~cm}^{-1}$.

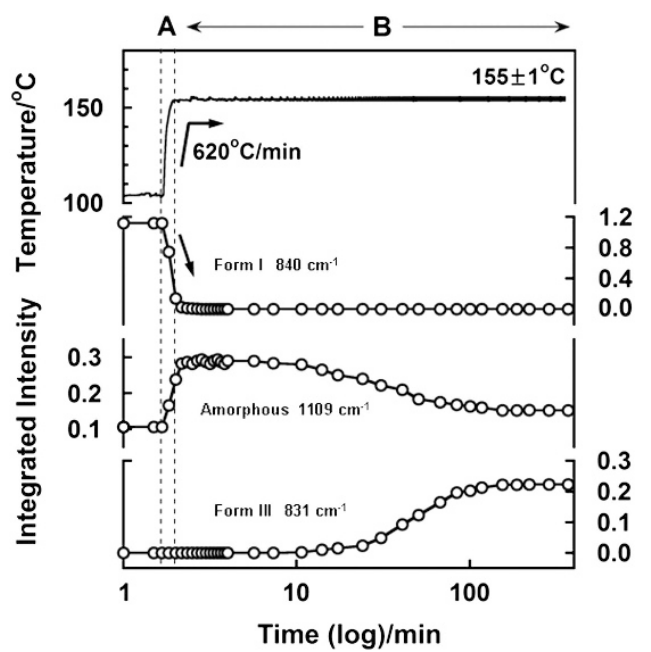

Figure 3 Time dependence of the infrared absorbance estimated for the characteristic bands of crystalline forms I and III in the isothermal heating process. 
above, we proposed in previous studies ${ }^{15,16}$ that form I transformed directly into form III (or V) in the temperature region immediately below the melting point through the temperature-dependent measurements of the infrared spectra and X-ray diffraction. As is likely the case for general polymers, the transition range can be so broad for the PVDF sample that it overlaps with the melting region, causing the transition behavior to be quite ambiguous. The temperature-dependent experimental data misled us, causing us to conclude that the direct solid-state phase transition is the appropriate phase-transition mechanism. Figure 4 shows the schematic illustration of the conformational change and the packing structure change of PVDF form I to form III (or form V). The trans-zigzag chains transform to the gauche-type form. The chain packing is also changed, where form III takes the configuration of a polar crystal with the chains packed in parallel, while form $\mathrm{V}$ (or anti-parallel form III) takes the configuration of the nonpolar crystal structure.

Note that the sample used in the present experiment was that cast from the solution, which was not treated under the high electric field to yield the highly poled film. The PVDF form I sample used in the previous studies ${ }^{15,16}$ was that treated under a high electric field or the poled sample. According to the observation by transmission electron microscopy, the VDF-TrFE copolymer with $75 \%$ VDF content resulted in a large crystal region after being treated by the poling process, which demonstrated a ferroelectric-to-paraelectric phase transition in a much higher temperature region compared with the case of the non-poled sample. ${ }^{34}$ The same difference between highly poled and non-poled samples might apply for PVDF form I, which is a possibility that will be investigated in the future.

\section{Phase transition of TPI}

A temperature-jump experiment similar to that described above for PVDF was performed for a TPI sample. Before the temperature-jump

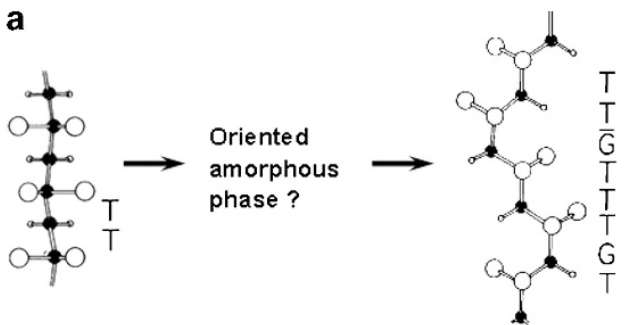

b

Form I
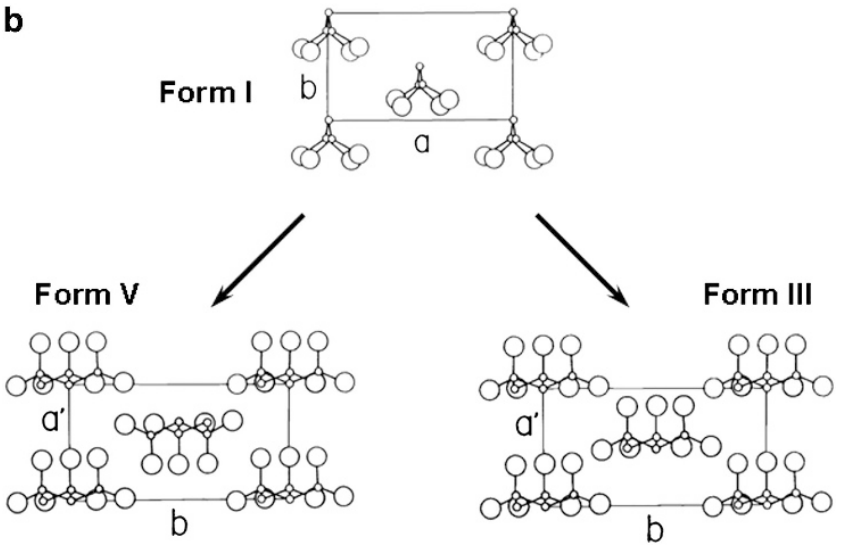

Figure 4 Illustration of the phase transition of PVDF form I in the hightemperature region close to the melting point. The crystalline forms III and $V$ are different in the packing mode of the molecular chains of TTTGTTTG conformation. experimental results are described, we first describe the investigation of the transition behavior during the continuous (or stepwise) heating process. The unoriented $\beta$-sample of TPI was heated continuously, during which time the $\mathrm{X}$-ray diffraction and infrared spectral measurements were performed, as shown in Figures 5 and 6, respectively. During the heating process that began from room temperature, the X-ray diffraction peaks intrinsic to the $\beta$-form decreased in intensity at approximately $50^{\circ} \mathrm{C}$. The broad amorphous halo and the weak but sharp $\alpha$ form peaks increased as the $\beta$-form peaks decreased. A similar transition was also observed in the infrared spectra. The integrated intensities of the X-ray diffraction and infrared peaks are plotted against temperature for the $\alpha$ - and $\beta$-forms, as well as for the amorphous phase, as shown in Figure 7. The X-ray and IR band intensities of the $\beta$-form decreased in the temperature region of $45-55^{\circ} \mathrm{C}$, where the infrared bands of the amorphous and $\alpha$-form increased in intensity. With further heating, the $\alpha$-peaks decreased in intensity and eventually disappeared.

From the data during continuous heating, the appearance of the amorphous phase and the $\alpha$-form were observed to occur in almost the same temperature region (II and III) where the endothermic peak was detected in the DSC thermogram. From these observations, we conclude that it is difficult to determine whether the $\beta$-to- $\alpha$ transition mechanism is due to melt-recrystallization or a solid-to-solid transition.

The phase transition for an oriented sample of TPI during the heating process was also investigated, although the data are not presented here. Similar to the case of the unoriented sample, the twodimensional X-ray diffraction pattern and polarized infrared spectra of the $\beta$-form decreased, and the peaks originating from the amorphous and $\alpha$-forms were observed to appear in the same temperature region. All of these data for the oriented sample still do not elucidate the plausible transition mechanism. Note that the orientation of the crystalline phase remained unchanged even after the completion of the phase transition from the $\beta$ - to the $\alpha$-form.

We conclude that it is difficult to choose the best candidate for the $\beta$-to- $\alpha$ transition mechanism on the basis of the experimental data of WAXD and FTIR spectra collected for both the unoriented and oriented samples in the stepwise (or continuous) heating process.

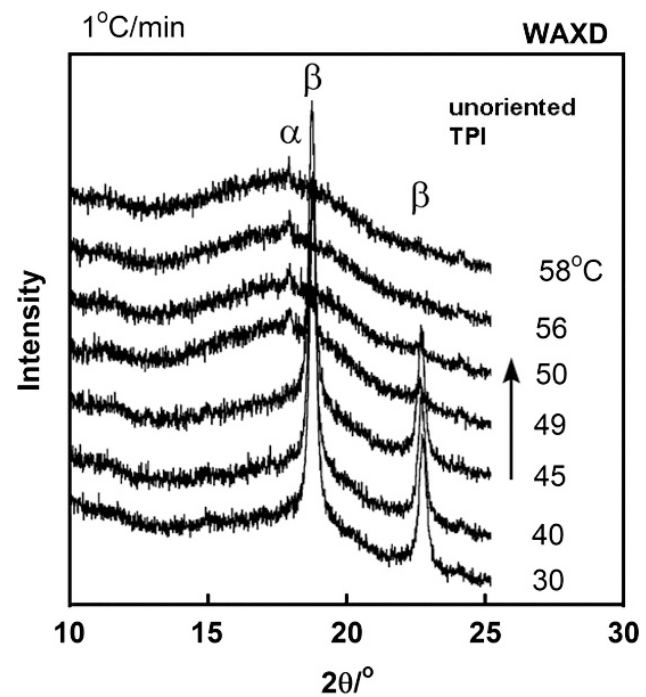

Figure 5 Temperature dependence of the X-ray diffraction pattern measured for the unoriented TPI $\beta$-sample during the stepwise heating at an average rate of $1^{\circ} \mathrm{C} \mathrm{min}^{-1}$. 
FTIR

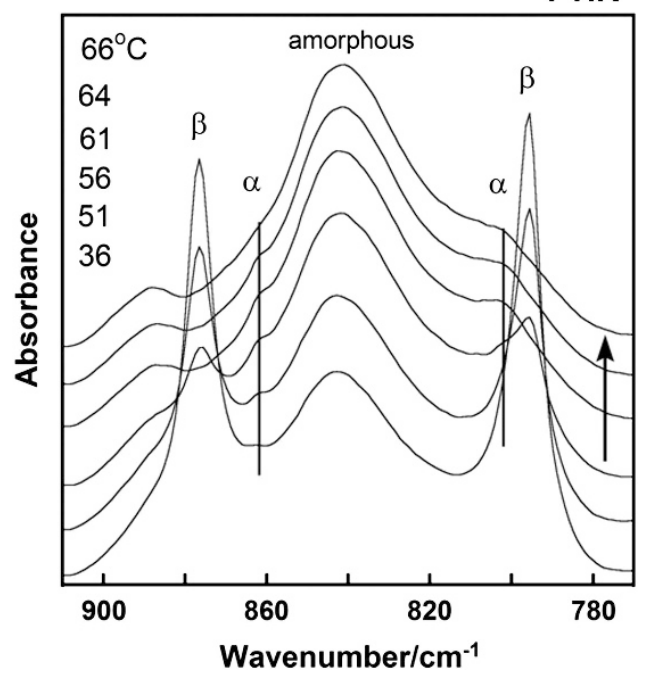

Figure 6 Temperature dependence of the infrared spectra measured for the unoriented TPI $\beta$-sample during the stepwise heating at an average rate of $1{ }^{\circ} \mathrm{C} \min ^{-1}$.

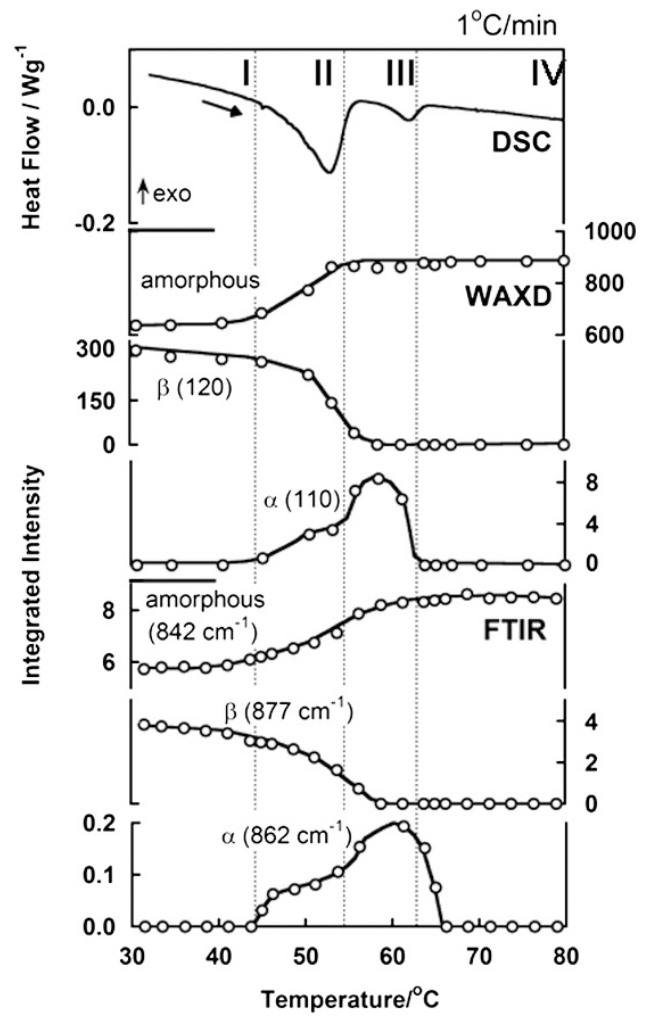

Figure 7 Temperature dependence of the X-ray diffraction intensity and the infrared absorbance estimated for the unoriented TPI $\beta$-form during the heating process.

To provide more insight, time-resolved measurements of the FTIR spectra and WAXD patterns were performed during a temperature jump. Figures $8 \mathrm{a}$ and $\mathrm{b}$ show the FTIR spectral changes measured for the unoriented $\beta$-sample during the temperature-jump process from an initial temperature of 27 to $47^{\circ} \mathrm{C}$ and $50^{\circ} \mathrm{C}$, respectively. Figure 9 shows the corresponding intensity data estimated for the IR bands of the $\beta$-form, amorphous phase, and $\alpha$-form. For example, the a $47^{\circ} \mathrm{C}$

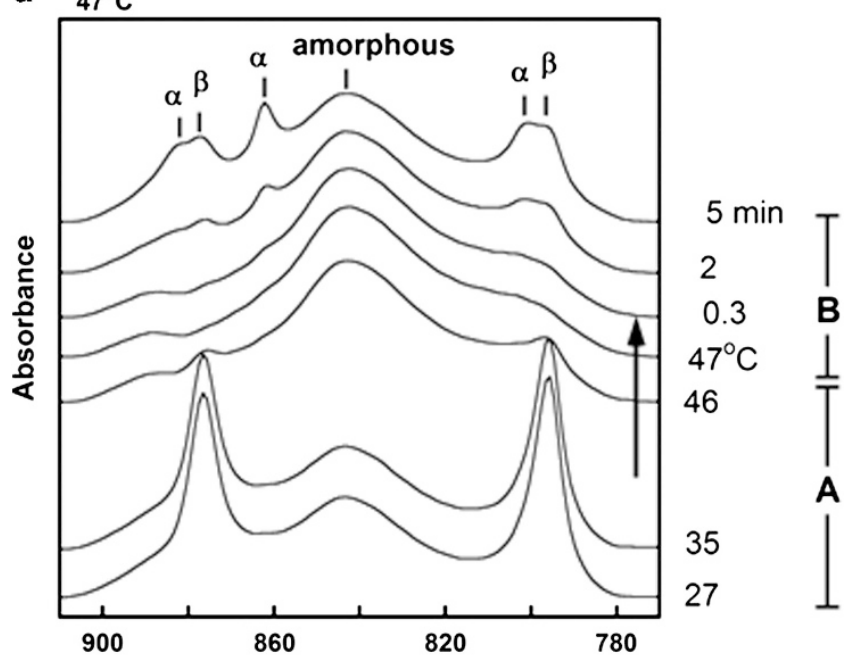

b $50^{\circ} \mathrm{C}$

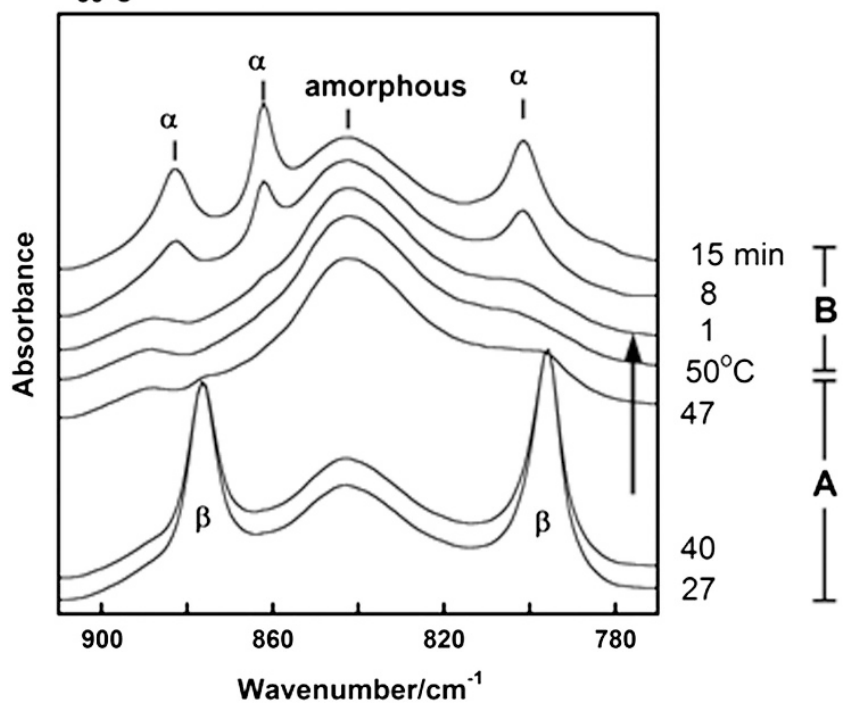

Figure 8 Time dependence of the infrared spectra of TPI measured in the isothermal heating process at (a) $47^{\circ} \mathrm{C}$ and (b) $50^{\circ} \mathrm{C}$.

$877 \mathrm{~cm}^{-1}$ band intrinsic to the $\beta$-form started to decrease in intensity during the temperature jump to $47^{\circ} \mathrm{C}$ (process $\mathrm{A}$ ). The amorphous band at $842 \mathrm{~cm}^{-1}$ increased the intensity during process A. After the temperature reached $47^{\circ} \mathrm{C}$, the amorphous band intensity started to decrease, just when the bands of the $\alpha$-form began to appear and slowly increase in intensity (process B). Similar observations were also made for the temperature jump to $50^{\circ} \mathrm{C}$, although the transition rate was relatively lower compared with the case of the jump to $47^{\circ} \mathrm{C}$. All of these experimental data clearly indicate that the $\beta$-form initially transforms to the amorphous state and subsequently recrystallizes into the $\alpha$-form.

As indicated above, the chain orientation remained constant through the transition from the $\beta$-form to the amorphous phase and eventually to the $\alpha$-form. This constant orientation was confirmed in the time-resolved measurements of the two-dimensional WAXD pattern in the temperature-jump experiment, as shown in Figure 10. (The WAXD measurement was performed with the oriented sample fixed to the metal holder so that the sample did 

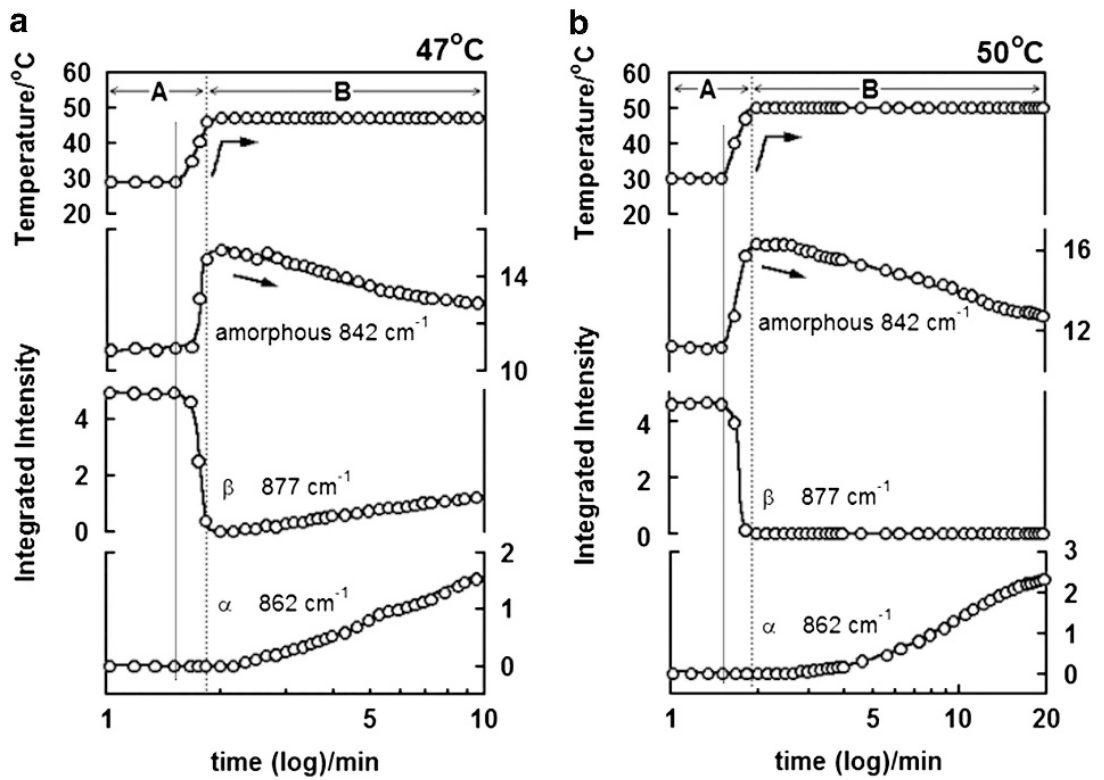

Figure 9 Logarithmic plot of the time dependence of the integrated infrared band intensity estimated for the amorphous phase and the $\alpha$ - and $\beta$-forms of TPI during the temperature-jump process: (a) from 27 to $47^{\circ} \mathrm{C}$ and (b) from 27 to $50{ }^{\circ} \mathrm{C}$.

not shrink during the heating process near the melting region. As a result, the melting point was shifted upward by a few degrees, but the crystalline orientation was not significantly affected because a similar phenomenon was also detected for the experiment performed under tension-free conditions, although the resulting $\alpha$-form exhibited a much lower degree of orientation).

As shown in Figure 10, the X-ray diffraction spots remained during the transition process from the $\beta$-form to the $\alpha$-form via the amorphous phase, although the chain orientation during the $\alpha$-form formation became slightly worse, as indicated by the longer arc shape of the X-ray diffraction spots. The preservation of the chain orientation between the $\beta$ - and $\alpha$-forms indicates that the intermediate amorphous phase must also be oriented. In fact, the amorphous halo indicated by an arrow in Figure 10 did not exhibit an isotropic ring pattern; instead, the data exhibited anisotropy in the relative intensity along the azimuthal direction, indicating the existence of an oriented amorphous state. In other words, the intermediate phase appearing during the phase transition is not in a simple isotropic amorphous state, but it might be in the form of an oriented liquid-crystalline state. According to the X-ray structure analysis, the chain conformations of the $\beta$ - and $\alpha$-forms are described as follows (see Figure 11):

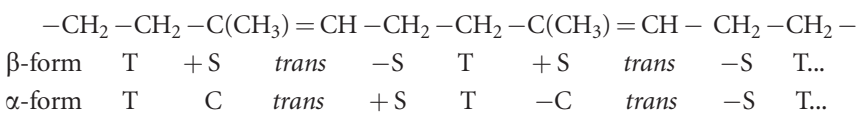

As understood from the large difference in the torsional angles of the skeletal $\mathrm{C}-\mathrm{C}$ bonds (for example, $-\mathrm{S} \rightarrow+\mathrm{S}$ and $+\mathrm{S} \rightarrow \pm \mathrm{C}$ ), it is difficult to imagine the direct and smooth transformation of the chain conformation between these two phases. This difference in torsional angles suggests that any conformational disorder for the induction of the conformational change between the two crystalline phases must occur in the intermediate phase. During this conformational disordering process, the molecular chain orientation must remain unchanged. A proposed transition scheme is illustrated in Figure 11. As indicated above, the intermediate phase might be in a type of oriented liquid-crystalline state, consisting of the oriented chains with some conformational disorder.

This structural transitional scheme leads us to predict the two-stage change of the mechanical property of TPI in the high-temperature region. To test this prediction, the Young's modulus of an unoriented $\beta$-form sample was measured during the heating process using DVA equipment. (Ideally, an oriented sample should be used, but the oriented $\beta$-sample was easily broken in the high-temperature region close to the melting point, and measuring the temperature change of the Young's modulus was impossible).

Figure 12 shows the temperature dependence of $E^{\prime}$, or a real part of Young's modulus, and the corresponding $\tan (\delta)$. The $E^{\prime}$ and $\tan (\delta)$ changed at two stages in the $\beta$-to-amorphous- $\alpha$ phase-transition region. If a purely crystalline TPI $\beta$-form is used in the DVA measurement, although it is impossible to achieve for this polymer, we should observe the sharp changes of $E^{\prime}$ in three temperature regions: (i) a sharp reduction of $E^{\prime}$ in the $\beta$-to-amorphous transition, (ii) an increase in $E^{\prime}$ during the recrystallization process from the amorphous phase to the $\alpha$-crystal and (iii) a decrease of $E^{\prime}$ due to the melting of the $\alpha$-crystal. In the actual experiment, the $E^{\prime}$ exhibited two deflections at the two temperatures of 55.8 and $63.1^{\circ} \mathrm{C}$, corresponding to the transitions mentioned above, although the overlapping of these phase transitions cause the curves to be more or less ambiguous, as seen in the temperature dependences of X-ray diffraction and infrared spectral data (Figures 5-7). In the experiment, the sample was unoriented; thus, the $E^{\prime}$ is an average of the Young's moduli in all of the directions. Nevertheless, the data clearly reflected the structural changes in the crystalline and amorphous phases because the remarkable structural change in the crystal lattice occurs in all of the directions, including the directions parallel and perpendicular to the chain axis.

\section{CONCLUSION}

Clarification of the phase-transition process between the crystalline forms of polymers is important for the scientific establishment of the transition mechanism as well as for the improvement of the physical 


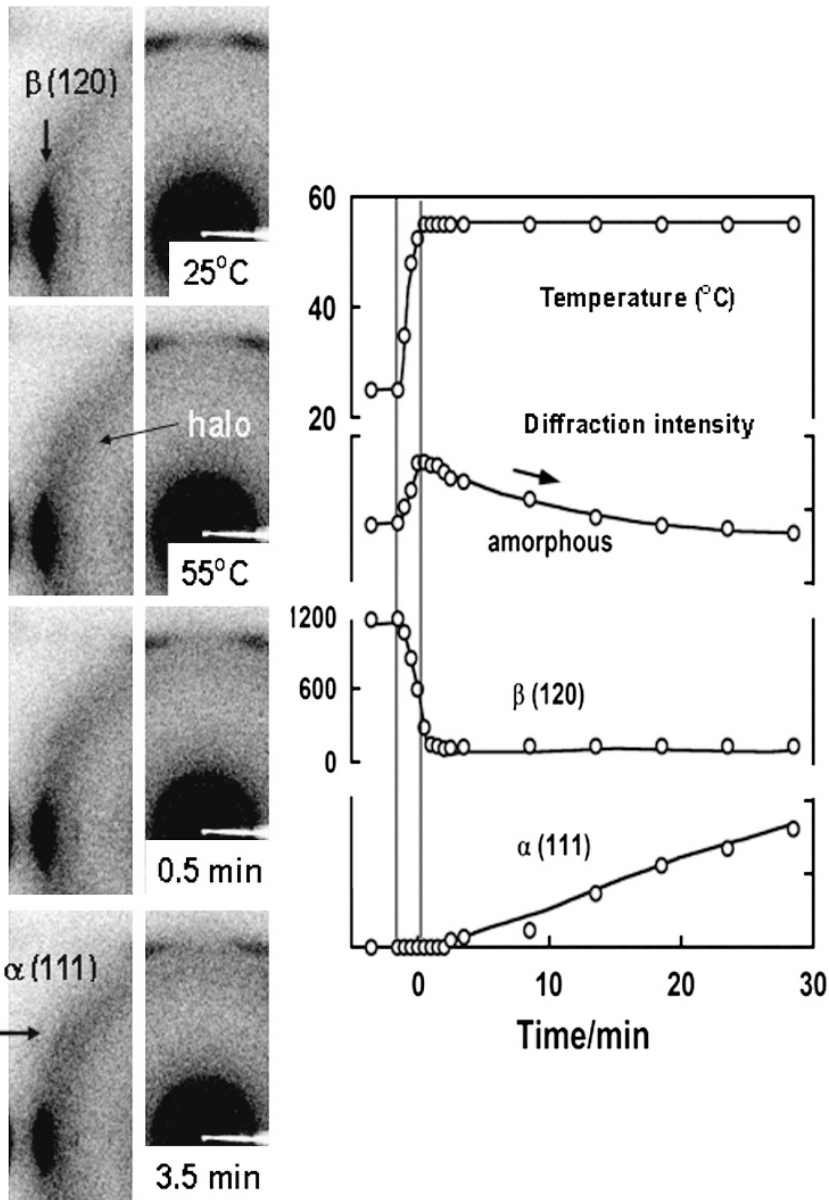

Figure 10 (left) Temperature and time dependences of the two-dimensional WAXD pattern of an oriented TPI sample measured during the isothermal heating process at $155^{\circ} \mathrm{C}$. The first two patterns were measured during the rapid heating process, and the others were during the isothermal process as a function of time at $155^{\circ} \mathrm{C}$. Note that the amorphous halo indicated by an arrow exhibited an anisotropic intensity distribution along the azinuthal direction, indicating the existence of an oriented amorphous or liquidcrystalline state in the sample. (right) The time dependence of the temperature and the integrated intensity of the observed X-ray diffraction peaks of the $\beta$-form, the amorphous (liquid-crystalline) phase and the $\alpha$-form.

properties developed during polymer processing. In many reports published so far on the phase transition of crystalline polymers, the $\mathrm{X}$-ray diffraction and vibrational spectra were measured in the continuous (or stepwise) change of temperature. Because the transition temperature region is appreciably wide and the X-ray diffraction spots are broad, the transition process is ambiguous, making it difficult to clearly judge the transition behavior. The time-resolved measurement of X-ray diffraction and FTIR spectra during the course of the temperature-jump process from the low-temperature phase to the high-temperature phase allowed us to identify the mechanism of the phase-transition process. This time-resolved technique was successfully applied to the phase-transition phenomenon of the ferroelectric PVDF form I and that of TPI between the $\beta$ - and $\alpha$-forms. In both of these cases, the transition did not occur directly but via an intermediate phase, which is possibly an oriented liquidcrystalline state.

Identifying the correct mechanism between the possibilities of the direct solid-to-solid transition and the melt-recrystallization process is

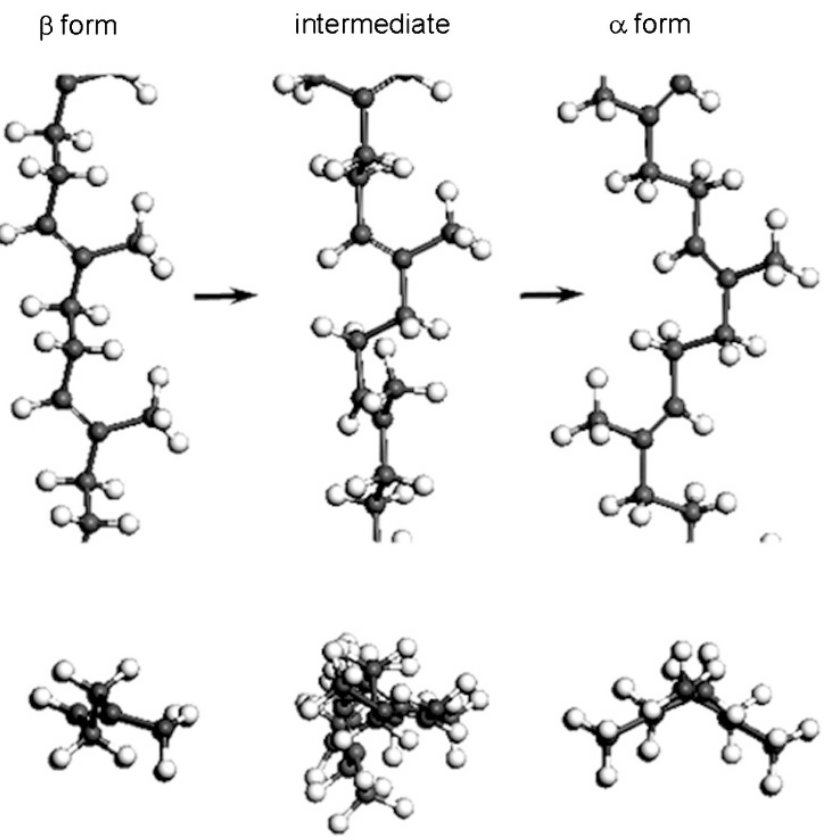

Figure 11 Schematic illustration of the structural transformation from the $\beta$-form to the $\alpha$-form via the intermediate liquid-crystalline phase.

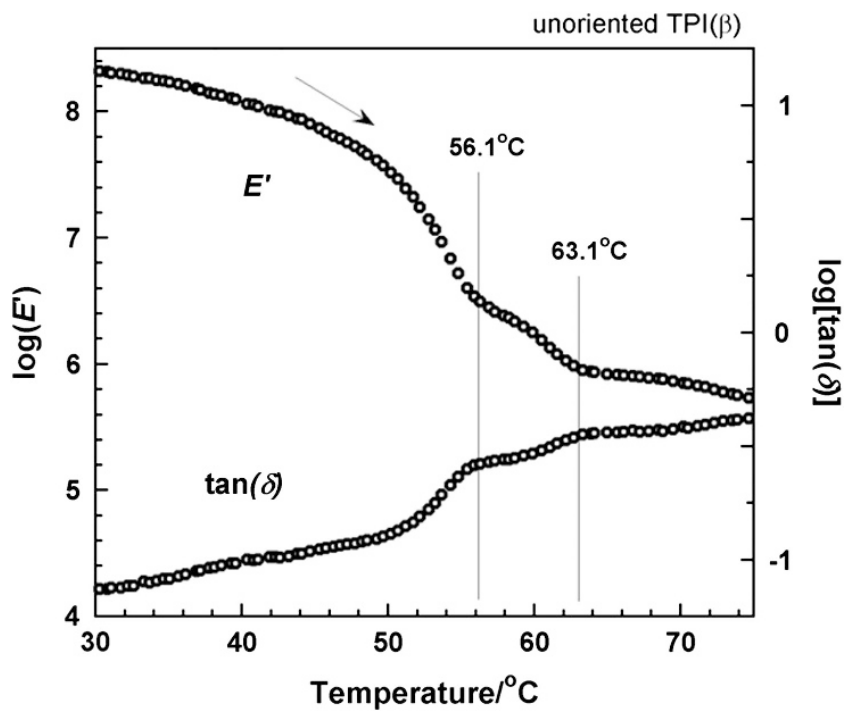

Figure 12 Temperature dependence of $E$ and $\tan (\delta)$ measured during the heating process of the unoriented TPI $\beta$-sample.

very important for the theoretical interpretation of the phasetransition mechanism. This fact can also be said for the interpretation of the temperature dependence of the mechanical properties, as exemplified by the case of TPI. As mentioned in the introductory session, there are many types of phase transitions for the various types of crystalline polymers, among which some cases might have been incorrectly interpreted. The kinetic technique reported here may help correct these misunderstandings.

\section{ACKNOWLEDGMENTS}

We thank Dr Tadaoki Ii of IT Keisoku Co. Ltd., Japan, for his DVA measurement of the TPI sample. This study was supported financially by MEXT 'Strategic Project to Support the Formation of Research Bases at Private Universities (2010-2014)'. 
1 Sperati, C. A. \& Starkweather, Jr H. W. Fluorine-containing polymers II polytetrafluoroethylene. Adv. Polym. Sci. 2, 465-495 (1961).

2 Clark, E. S. \& Muus, L. T. Partial disordering and crystal transitions in polytetrafluoroethylene. ZA. Krist 117, 119-127 (1962).

3 Brill, R. J. J. Prakt. Chem. 161, 49 (1942).

4 Yoshioka, Y., Tashiro, K. \& Ramesh, C. Structural change in the Brill transition of nylon $\mathrm{m} / \mathrm{n}$ (2) conformational disordering as viewed from the temperature-dependent infrared spectral measurements. Polymer (Guildf) 44, 6407-6417 (2003).

5 Yoshioka, Y. \& Tashiro, K. Structural change in the Brill transition of nylon $\mathrm{m} / \mathrm{n}$ (1) nylon 10/10 and its model compounds. Polymer (Guildf) 44, 7007-7019 (2003).

6 Tashiro, K. Structural phase transition of aliphatic nylons viewed from the WAXD/SAXS and vibrational spectral measurements and molecular dynamics calculation. Chinese $J$ Polym. Sci. 25, 73-82 (2007)

7 Chau, K. W., Yang, Y. C. \& Geil, P. H. Tetragonal-twinned hexagonal crystal phase transformation in polybutene-1. J. Mat. Sci. 21, 3002-3014 (1986).

8 Gohil, R. M., Miles, M. J. \& Petermann, J. On the molecular mechanism of the crystal transformation (tetragonal-hexagonal) in polybutene-1. J. Macromol. Sci. Part B: Phys. 21, 189-201 (1982).

9 Jakeways, R., Ward, I. M., Wilding, M. A., Hall, I. H., Desborough, I. J. \& Pass, M. G. Crystal deformation in aromatic polyesters. J. Polym. Sci. Polym. Phys. Part B 13, 799-813 (1975)

10 Tashiro, K., Nakai, Y., Kobayashi, M. \& Tadokoro, H. Solid-state transition of poly(butylenes terephthalate) induced by mechanical deformation. Macromolecules 13, 137-145 (1980).

11 Takahashi, Y., Sumita, I. \& Tadokoro, H. Structural studies of polyethers IX. Planar zigzag modification of poly(ethylene oxide). J. Polym. Sci. Polym. Phys. Ed. 11, 2113-2122 (1973).

12 Lovinger, A. J. Recent development in the structure, properties, and applications of ferroelectric polymers. Jpn. J. Appl. Phys. Suppl. 24, 18-22 (1985).

13 Tashiro, K. \& Kobayashi, M. Structural phase transition in ferroelectric fluorine polymers: X-ray diffraction and infrared/Raman spectroscopic study. Phase Transitions 18, 3-4 (1989).

14 Tashiro, K. In Crystal Structure and Phase Transition of PVDF and Related Copolymers, Ferroelectric Polymers: Chemistry, Physics, and Technology (Nalwa, H. S. ed.) 63-182 (Marcel Dekker Inc., New York, 1995).

15 Tashiro, K., Takano, K., Kobayashi, M., Chatani, Y. \& Tadokoro, H. Phase transition at a temperature immediately below the melting point of poly(vinylidene fluoride) form I: a proposition for the ferroelectric curie point. Polymer (Guildf) 24, 199-204 (1983).

16 Tashiro, K., Takano, K., Kobayashi, M., Chatani, Y. \& Tadokoro, H. A preliminary X-ray study on ferroelectric phase transition of poly(vinylidene fluoride) crystalline form I. Polym. Bull. 10, 464-469 (1983).
17 Lovinger, A. J., Davis, G. T., Cais, R. E. \& Kometani, J. M. On the Curie temperature of poly(vinylidene fluoride). Macromolecules 19, 1491-1494 (1986).

18 Ohigashi, H. Piezoelectric polymers - materials and manufacture. Jpn. J. Appl. Phys. Suppl. 24, 23-27 (1985).

19 Lovering, E. G. \& Wooden, D. C. Transition in trans-1,4-polyisoprene. J. Polym. Sci. 7 1639-1649 (1969).

20 Schilder, H., Goodman, A. \& Aldrich, W. The thermomechanical properties of Guttapercha III. Determination of phase transition temperatures for Gutta-percha. Oral Surg. 38, 109-114 (1974).

21 Rootare, H. M. \& Powers, J. M. Determination of phase transitions in gutta-percha by differential thermal analysis. J. Dent. Res. 56, 1453-1462 (1977).

22 Fischer, E. \& Henderson, J. F. Study of crystallization kinetics of trans-1,4-polyisoprene. J. Polym. Sci. 5, 377-390 (1967).

23 Martuscelli, E. Annealing behaviour of melt crystallized trans-1,4-polyisoprene. Die Makromolekulare Chemie 151, 159-168 (1972).

24 Hardin, I. R. \& Yeh, G. S. Y. Strain-induced crystallization and reversible phenomena in transpolyisoprone II. Xray studies. J. Macromol. Sci. Part B 7, 393-409 (1973).

25 Yeh, G. S. Y. Thermal reversibility of crystal surface structure and its contribution to degree of crysallinity in natural rubber and trans-polyisoprene. J. Macromol. Sci. Part B 8, 241-257 (1973).

26 Lando, J. B., Olf, H. G. \& Peterlin, A. NMR and X-ray determination of the structure of poly(vinylidene fluoride). J. Polym. Sci.Part A 1, 941-951 (1966).

27 Hasegawa, R., Takahashi, Y., Chatani, Y. \& Tadokoro, H. Crystal structure of three crystalline forms of poly(vinylidene fluoride). Polym. J. 3, 591-599 (1972).

28 Kobayashi, M., Tashiro, K. \& Tadokoro, H. Molecular vibrations of three crystalline forms of poly(vinylidene fluoride). Macromolecules 8, 158-171 (1975).

29 Ratri, P. J., Tashiro, K. \& Iguchi, M. Experimentally- and theoretically-evaluated ultimate 3-dimensional elastic constants of trans-1,4-polyisoprene $\alpha$ and $\beta$ crystalline forms on the basis of the newly-refined crystal structure information. Polymer (Guildf) 53, 3548-3558 (2012).

30 Takahashi, Y. \& Tadokoro, H. Crystal structure of form III of poly(vinylidene fluoride). Macromolecules 13, 1317-1318 (1980).

31 Tashiro, K., Kobayashi, M. \& Tadokoro, H. Vibrational spectra and disorder-order transition of poly(vinylidene fluoride) form III. Macromolecules 14, 1757-1764 (1981).

32 Lovinger, A. J. Annealing of poly(vinylidene fluoride) and formation of a fifth phase. Macromolecules 14, 40-48 (1982).

33 Tashiro, K., Itoh, Y., Kobayashi, M. \& Tadokoro, H. Polarized raman spectra and LO-TO splitting of poly(vinylidene fluoride) crystalline form I. Macromolecules 18, 2600-2606 (1985).

34 Ohigashi, H., Kagami, N. \& Li, G. R. Formation of ferroelectric domains in a copolymer (PVDF-TrFE). J. Appl. Phys. 71, 506-508 (1992). 